\title{
The homotopy analysis method and the Liénard equation
}

\author{
S. Abbasbandy ${ }^{\mathrm{a}, \mathrm{b}, *}$, J.L. López ${ }^{\mathrm{c}}$, R. López-Ruiz ${ }^{\mathrm{d}}$ \\ ${ }^{a}$ Department of Mathematics, Science and Research Branch, Islamic Azad \\ University, Tehran, 14778, Iran \\ ${ }^{\mathrm{b}}$ Department of Mathematics, Imam Khomeini International University, Ghazvin, \\ 34149-16818, Iran \\ ${ }^{\mathrm{c}}$ Department of Mathematical and Informatics Engineering, Universidad Pública \\ de Navarra, 31006-Pamplona, Spain \\ ${ }^{\mathrm{d}}$ Department of Computer Science and BIFI, Universidad de Zaragoza, \\ 50009-Zaragoza, Spain
}

\begin{abstract}
In this work, Liénard equations are considered. The limit cycles of these systems are studied by applying the homotopy analysis method. The amplitude and frequency obtained with this methodology are in good agreement with those calculated by computational methods. This puts in evidence that the homotopy analysis method is an useful tool to solve nonlinear differential equations.
\end{abstract}

Key words: Liénard equation; Homotopy analysis method; Limit cycles PACS numbers: 02.30.Hq, 02.30.Mv, 02.60.Lj

AMS Classification: 34C07, 65L80

\section{Introduction}

A generalization of the van der Pol oscillator is the classical Liénard differential equation,

$$
\ddot{x}(t)+\epsilon f(x) \dot{x}(t)+x(t)=0, \quad t \geq 0,
$$

with $\epsilon$ a real parameter and $f(x)$ any real function. The dot denotes the derivative with respect to time $t$. The periodic solutions of this system are called limit cycles [1]. For instance, when $f(x)=x^{2}-1$ (van der Pol oscillator), Eq. 1.1 displays

* Corresponding author.

Email address: abbasbandy@yahoo.com (S. Abbasbandy). 
a limit cycle whose uniqueness and non-algebraicity has been shown for the whole range of the parameter $\epsilon$ [2]. Its behavior runs from near-harmonic oscillations when $\epsilon \rightarrow 0$ to relaxation oscillations when $\epsilon \rightarrow \infty$, making it a good model for many practical situations 3 . Other partial results on the number and form of limit cycles in Liénard systems are scattered in the literature [4]. When $f(x)$ is a polynomial of degree $N=2 n+1$ or $2 n$, with $n$ a natural number, Lins, Melo and Pugh have conjectured (LMP-conjecture) that the maximum number of limit cycles allowed is just $n$ [5]. It is true if $N=2$, or $N=3$ or if $f(x)$ is even and $N=4[5,6]$. Also, there are strong arguments for claiming its truth in the strongly nonlinear regime $(\epsilon \rightarrow \infty)$ when $f(x)$ is an even polynomial [7] and recently in the weakly nonlinear regime $(\epsilon \rightarrow 0)$ for even $f(x)$ [8]. However, this conjecture has been recently shown [9] to have counterexamples for $n \geq 3$ when $f(x)$ is not even. In particular, it has been found a polynomial $f(x)$ of degree 6 such that the associated Liénard equation has at least 4 limit cycles [9]. Thus, there are no general results about the limit cycles when $f(x)$ is a polynomial of degree greater than 5 neither, in general, when $f(x)$ is an arbitrary real function [10].

Apart from the classical perturbative techniques that can be applied in the weakly nonlinear regime [11,12,13], different non-perturbative approaches allowing to obtain information on the number of limit cycles and their location in phase space have been proposed in the last years. A method that gives a sequence of algebraic approximations to the equation of each limit cycle can be found in [10], and a variational method showing that limit cycles correspond to relative extrema of certain functionals is explained in [14. Here, we are interested in the application of another non-perturbative technique, the homotopy analysis method (HAM), to this problem. Liao [15,16] has developed this purely analytic technique to solve nonlinear problems in science and engineering. The HAM has been applied successfully to many nonlinear problems such as free oscillations of self-excited systems [17], the generalized Hirota-Satsuma coupled KdV equation [18, heat radiation [19], finding the root of nonlinear equations [20], finding solitary-wave solutions for the fifth-order $\mathrm{KdV}$ equation [21], finding solitary wave solutions for the Kuramoto-Sivashinsky equation [22], finding the solitary solutions for the Fitzhugh-Nagumo equation [23], boundary-layer flows over an impermeable stretched plate [24], unsteady boundarylayer flows over a stretching flat plate [25], exponentially decaying boundary layers [26], a nonlinear model of combined convective and radiative cooling of a spherical body [27], and many other problems (see [28,29,30,31,32,33,34,35,36], for example).

In this paper, we are interested in applying the HAM to Liénard equation (1.1) in order to obtain good approximations to the amplitude and shape of its limit cycles. These calculations are explained in Section 2. The validity of the method (for arbitrary $\epsilon$ ) is shown for the different particular cases analyzed in Section 3. Last section includes our conclusions. 


\section{HAM applied to Liénard equations}

In general, the limit cycles of (1.1) contain two important physical parameters, i.e. the frequency $\omega$ and the amplitude $a$. So, without loss of any generality, consider such initial conditions:

$$
x(0)=a, \quad \dot{x}(0)=0,
$$

where $a>0$ is the amplitude of the limit-cycle.

Let $\tau=\omega t$ denotes a new time scale, with $\omega>0$. Under the transformation

$$
\tau=\omega t, \quad x(t)=a u(\tau)
$$

the original Eq. (1.1) and its initial conditions (2.1) become

$$
\omega^{2} u^{\prime \prime}(\tau)+\epsilon \omega f(a u) u^{\prime}(\tau)+u(\tau)=0,
$$

and

$$
u(0)=1, \quad u^{\prime}(0)=0,
$$

respectively, where the prime denotes the derivative with respect to $\tau$.

The limit-cycles of (2.3) are periodic motions with period $T=2 \pi / \omega$ and thus $u(\tau)$ can be expressed by

$$
u(\tau)=\sum_{m=0}^{+\infty}\left[\alpha_{m} \sin (m \tau)+\beta_{m} \cos (m \tau)\right]
$$

where $\alpha_{m}$ and $\beta_{m}$ are coefficients to be determined. According to the rule of solution expression denoted by (2.5) and the boundary conditions (2.4), it is natural to choose

$$
u_{0}(\tau)=\cos (\tau)
$$

as the initial approximation to $u(\tau)$. Let $\omega_{0}$ and $a_{0}$ denote the initial approximations of the frequency $\omega$ and the amplitude $a$, respectively.

We define an auxiliary linear operator $\mathcal{L}$ by

$$
\mathcal{L}[\phi(\tau ; p)]=\omega_{0}^{2}\left(\frac{\partial^{2}}{\partial \tau^{2}}+1\right) \phi(\tau ; p)
$$

with the property

$$
\mathcal{L}\left[C_{1} \sin (\tau)+C_{2} \cos (\tau)\right]=0,
$$

where $C_{1}$ and $C_{2}$ are constants, and $p$ is a parameter explained below.

From (2.3) we define a nonlinear operator

$$
\mathcal{N}[\phi(\tau ; p), A(p), \Omega(p)]=\Omega^{2}(p) \frac{\partial^{2} \phi(\tau ; p)}{\partial \tau^{2}}+\epsilon \Omega(p) f(A(p) \phi(\tau ; p)) \frac{\partial \phi(\tau ; p)}{\partial \tau}+\phi(\tau ; p),
$$


and then construct the homotopy

$$
\mathcal{H}[\phi(\tau ; p), A(p), \Omega(p)]=(1-p) \mathcal{L}\left[\phi(\tau ; p)-u_{0}(\tau)\right]-h p \mathcal{N}[\phi(\tau ; p), A(p), \Omega(p)]
$$

where $h$ is a nonzero auxiliary parameter. Setting $\mathcal{H}[\phi(\tau ; p), A(p), \Omega(p)]=0$, we have the zero-order deformation equation

$$
(1-p) \mathcal{L}\left[\phi(\tau ; p)-u_{0}(\tau)\right]=h p \mathcal{N}[\phi(\tau ; p), A(p), \Omega(p)]
$$

subject to the boundary conditions

$$
\phi(0 ; p)=1,\left.\quad \frac{\partial \phi(\tau ; p)}{\partial \tau}\right|_{\tau=0}=0
$$

where $p \in[0,1]$ is an embedding parameter. When the parameter $p$ increases from 0 to 1 , the solution $\phi(\tau ; p)$ varies from $u_{0}(\tau)$ to $u(\tau), A(p)$ varies from $a_{0}$ to $a$, and $\Omega(p)$ varies from $\omega_{0}$ to $\omega$. Assume that $\phi(\tau ; p), A(p)$ and $\Omega(p)$ are analytic in $p \in[0,1]$ and can be expanded in the Maclaurin series of $p$ as follows:

$$
\phi(\tau ; p)=\sum_{m=0}^{+\infty} u_{m}(\tau) p^{m}, \quad A(p)=\sum_{m=0}^{+\infty} a_{m} p^{m}, \quad \Omega(p)=\sum_{m=0}^{+\infty} \omega_{m} p^{m}
$$

where

$$
u_{m}(\tau)=\left.\frac{1}{m !} \frac{\partial^{m} \phi(\tau ; p)}{\partial p^{m}}\right|_{p=0}, \quad a_{m}=\left.\frac{1}{m !} \frac{\partial^{m} A(p)}{\partial p^{m}}\right|_{p=0}, \quad \omega_{m}=\left.\frac{1}{m !} \frac{\partial^{m} \Omega(p)}{\partial p^{m}}\right|_{p=0} .
$$

Notice that series (2.13) contain the auxiliary parameter $h$, which has influence on their convergence regions. Assume that $h$ is properly chosen such that all of these Maclaurin series are convergent at $p=1$. Hence at $p=1$ we have

$$
u(\tau)=u_{0}(\tau)+\sum_{m=1}^{+\infty} u_{m}(\tau), \quad a=a_{0}+\sum_{m=1}^{+\infty} a_{m}, \quad \omega=\omega_{0}+\sum_{m=1}^{+\infty} \omega_{m} .
$$

At the $M$ th-order approximation, we have the analytic solution of Eq. (2.3), namely

$$
u(\tau) \approx U_{M}(\tau)=\sum_{m=0}^{M} u_{m}(\tau), \quad a \approx A_{M}=\sum_{m=0}^{M} a_{m}, \quad \omega \approx \Omega_{M}=\sum_{m=0}^{M} \omega_{m} .
$$

The auxiliary parameter $h$ can be employed to adjust the convergence region of the series (2.14) in the homotopy analysis solution. By means of the so-called $h$ curve, it is straightforward to choose an appropriate range for $h$ which ensures the convergence of the solution series. As pointed out by Liao [16], the appropriate region for $h$ is indicated when $a$ and $\omega$ are horizontal segments when plotted versus $h$.

Differentiating Eqs. (2.11) and (2.12) $m$ times with respect to $p$, then setting $p=0$, and finally dividing by $m$ !, we obtain the $m$ th-order deformation equation

$$
\mathcal{L}\left[u_{m}(\tau)-\chi_{m} u_{m-1}(\tau)\right]=h R_{m}(\tau), \quad(m=1,2,3, \ldots),
$$


subject to the boundary conditions

$$
u_{m}(0)=0, \quad u_{m}^{\prime}(0)=0
$$

where $R_{m}(\tau)$ is defined by

$$
R_{m}(\tau)=\left.\frac{1}{(m-1) !} \frac{\partial^{m-1} \mathcal{N}[\phi(x ; p), A(p), \Omega(p)]}{\partial p^{m-1}}\right|_{p=0}
$$

and

$$
\chi_{m}=\left\{\begin{array}{l}
0, m \leq 1 \\
1, m>1
\end{array}\right.
$$

Notice that, both $a_{m}$ and $\omega_{m}$ remain unknown and due to the form of the solution (2.5) and definition (2.7), solutions of (2.15) and (2.16) should not contain the secular terms $\tau \sin (\tau)$ and $\tau \cos (\tau)$. It is easy to check that $\mathcal{L}[t \sin t]=2 \cos t$ and $\mathcal{L}[t \cos t]=-2 \sin t$, then the right-hand side term $R_{m}(\tau)$ of (2.17) should not contain the terms $\sin (\tau)$ and $\cos (\tau)$ in order to avoid the secular terms in the solution. Hence, the coefficients of $\sin (\tau)$ and $\cos (\tau)$ must be zero. If we rewrite

$$
R_{m}(\tau)=\sum_{i=1}^{\psi(m)}\left[c_{m, i} \cos (i \tau)+d_{m, i} \sin (i \tau)\right]
$$

then

$$
c_{m, i}=\frac{2}{\pi} \int_{0}^{\pi} R_{m}(\tau) \cos (i \tau) \mathrm{d} \tau, \quad d_{m, i}=\frac{2}{\pi} \int_{0}^{\pi} R_{m}(\tau) \sin (i \tau) \mathrm{d} \tau
$$

become zero when $i>\psi(m)$. Hence, we have two algebraic equations

$$
c_{m, 1}=0, \quad d_{m, 1}=0,
$$

which determine $a_{m-1}$ and $\omega_{m-1}$ for $m=1,2,3, \ldots$ The above two algebraic equations are often non-linear for $a_{0}$ and $\omega_{0}$ when $m=1$, but always linear in other case, as proved by Liao [15]. So, after solving $a_{m-1}$ and $\omega_{m-1}$, it is easy to gain the solution of (2.15) and (2.16) as

$$
u_{m}(\tau)=\chi_{m} u_{m-1}(\tau)+\sum_{i=2}^{\psi(m)} \frac{c_{m, i} \cos (i \tau)+d_{m, i} \sin (i \tau)}{\omega_{0}^{2}\left(1-i^{2}\right)}+C_{1} \cos (\tau)+C_{2} \sin (\tau)
$$

where the coefficients $C_{1}$ and $C_{2}$ are determined by (2.16). In this way, one can gain $a_{m-1}, \omega_{m-1}$ and $u_{m}(\tau)$ for $m=1,2,3, \ldots$, successively.

\section{Some examples}

In this section, the validity of the proposed method is illustrated by two examples. The limit cycles of different families of Liénard systems were studied in the weakly nonlinear regime [8,37. 
Example 1. The van der Pol oscillator is defined for $f(x)=x^{2}-1$. This system has a unique limit cycle, which is stable for $\epsilon>0$.

The corresponding perturbation approximation of the amplitude gives by a recursive algorithm the following formula

$$
a(\epsilon)=2+\frac{1}{96} \epsilon^{2}-\frac{1033}{552960} \epsilon^{4}+\frac{1019689}{55738368000} \epsilon^{6}+\mathcal{O}\left(\epsilon^{8}\right),
$$

reported in [8,13]. This analytical result agrees for small $\epsilon$ with the computational calculation of the 'exact' amplitudes calculated by a fourth-order Runge-Kutta method. Also, the expansion in $\epsilon$ of the frequency was obtained in [12] up to order $\mathcal{O}\left(\epsilon^{24}\right)$. For simplicity we give the expansion up to order $\mathcal{O}\left(\epsilon^{8}\right)$ :

$$
\omega(\epsilon)=1-\frac{\epsilon^{2}}{16}+\frac{17 \epsilon^{4}}{3072}+\frac{35 \epsilon^{6}}{884736}+\mathcal{O}\left(\epsilon^{8}\right) .
$$

Under transformation (2.2), Eq. (1.1) becomes

$$
\omega^{2} u^{\prime \prime}(\tau)+\epsilon \omega\left[a^{2} u^{2}(\tau)-1\right] u^{\prime}(\tau)+u(\tau)=0
$$

From (2.17), the term $R_{m}(\tau)$ in (2.15) becomes

$$
\begin{aligned}
R_{m}(\tau)= & \sum_{n=0}^{m-1} u_{m-1-n}^{\prime \prime}(\tau)\left(\sum_{j=0}^{n} \omega_{j} \omega_{n-j}\right)+u_{m-1}(\tau)-\epsilon \sum_{n=0}^{m-1} \omega_{n} u_{m-n-1}^{\prime}(\tau) \\
& +\epsilon \sum_{n=0}^{m-1}\left[\left(\sum_{i=0}^{m-1-n} \omega_{i} u_{m-n-i-1}^{\prime}(\tau)\right) \sum_{j=0}^{n}\left(\sum_{r=0}^{j} a_{r} a_{j-r}\right)\left(\sum_{s=0}^{n-j} u_{s}(\tau) u_{n-j-s}(\tau)\right)\right] .
\end{aligned}
$$

It is found that the frequency $\omega$ and the amplitude $a$ at the $M$ th-order of approximation can be expressed by

$$
\omega \approx \Omega_{M}=\omega_{0}+\sum_{i=1}^{M} \epsilon^{2 i} \sum_{j=i}^{M} \alpha_{M}^{i, j} h^{j}, \quad a \approx A_{M}=a_{0}+\sum_{i=1}^{M-1} \epsilon^{2 i} \sum_{j=i+1}^{M} \beta_{M}^{i, j} h^{j}
$$

respectively. So, $a_{0}$ and $\omega_{0}$ are obtained by solving (2.18) for $m=1$, i.e.

$$
c_{1,1}=\left(1-\omega_{0}^{2}\right)=0, \quad d_{1,1}=\epsilon \omega_{0}\left(1-\frac{1}{4} a_{0}^{2}\right)=0
$$

Hence, we have unique limit cycle by $\omega_{0}=1$ and $a_{0}=2$.

Note that results (3.5) contain the auxiliary parameter $h$. It is found that convergence regions of the approximation series are dependent upon $h$. The obtained results for amplitude are as follows 


$$
\begin{aligned}
& A_{1}=2, \\
& A_{2}=2+\frac{h^{2}}{96} \epsilon^{2}, \\
& A_{3}=2+\frac{h^{2} \epsilon^{2}}{32}+\frac{h^{3} \epsilon^{2}}{48}+\frac{h^{3} \epsilon^{4}}{768}, \\
& A_{4}=2+\frac{h^{2} \epsilon^{2}}{16}+\frac{h^{3} \epsilon^{2}}{12}+\frac{h^{4} \epsilon^{2}}{32}+\frac{h^{3} \epsilon^{4}}{192}+\frac{1847 h^{4} \epsilon^{4}}{552960}+\frac{h^{4} \epsilon^{6}}{6144},
\end{aligned}
$$

and for frequency are

$$
\begin{aligned}
& \Omega_{1}=1+\frac{h}{16} \epsilon^{2}, \\
& \Omega_{2}=1+\frac{h \epsilon^{2}}{8}+\frac{h^{2} \epsilon^{2}}{16}+\frac{3 h^{2} \epsilon^{4}}{512}, \\
& \Omega_{3}=1+\frac{3 h \epsilon^{2}}{16}+\frac{3 h^{2} \epsilon^{2}}{16}+\frac{h^{3} \epsilon^{2}}{16}+\frac{9 h^{2} \epsilon^{4}}{512}+\frac{37 h^{3} \epsilon^{4}}{3072}+\frac{5 h^{3} \epsilon^{6}}{8192}, \\
& \Omega_{4}=1+\frac{h \epsilon^{2}}{4}+\frac{3 h^{2} \epsilon^{2}}{8}+\frac{h^{3} \epsilon^{2}}{4}+\frac{h^{4} \epsilon^{2}}{16}+\frac{9 h^{2} \epsilon^{4}}{256}+\frac{37 h^{3} \epsilon^{4}}{768}+\frac{19 h^{4} \epsilon^{4}}{1024} \\
&+\frac{5 h^{3} \epsilon^{6}}{2048}+\frac{95 h^{4} \epsilon^{6}}{49152}+\frac{35 h^{4} \epsilon^{8}}{524288} .
\end{aligned}
$$

For example, for $h=-1$, the 10th-order approximation gives

$$
\begin{aligned}
A_{10}= & 2+\frac{\epsilon^{2}}{96}-\frac{1033 \epsilon^{4}}{552960}+\frac{1019689 \epsilon^{6}}{55738368000}+\frac{9835512276689 \epsilon^{8}}{157315969843200000}- \\
& \frac{58533181813182818069 \epsilon^{10}}{7326141789209886720000000}+\mathcal{O}\left(\epsilon^{12}\right), \\
\Omega_{10}= & 1-\frac{\epsilon^{2}}{16}+\frac{17 \epsilon^{4}}{3072}+\frac{35 \epsilon^{6}}{884736}-\frac{678899 \epsilon^{8}}{5096079360}+\frac{28160413 \epsilon^{10}}{2293235712000}+\mathcal{O}\left(\epsilon^{12}\right) .
\end{aligned}
$$

The general solution of Eq. (2.15) is

$$
u_{m}(\tau)=\hat{u}_{m}(\tau)+C_{1} \sin (\tau)+C_{2} \cos (\tau)
$$

where $C_{1}$ and $C_{2}$ are constants and $\hat{u}_{m}(\tau)$ is a particular solution of Eq. (2.15). Using (2.16), we can obtain the unknowns $C_{1}$ and $C_{2}$.

Our solution series contain the auxiliary parameter $h$. We can choose appropriate value of $h$ to ensure that the three solution series (2.14) converge. We can investigate the influence of $h$ on the convergence of $a$ and $\omega$ by plotting the curve of $a$ and $\omega$ versus $h$, as shown in Figs. 1 and 2. One can see on these plots that, for $\epsilon=1$, we have $-1.4 \leq h \leq-0.4$ and for $\epsilon=0.5$, we have $-1.4 \leq h \leq-0.2$. The comparison of the amplitude $a$ and the frequency $\omega$ at the 10th-order of approximation with the numerical results is as shown in Figs. 3 and 4 , where $h=-1,-\frac{2}{3}$ and $-\frac{1}{3}$. However, as $h$ is negative and close to zero, the convergence region becomes larger 
and larger. Note that, one has a great freedom to choose the auxiliary parameter $h$. Certainly, this can be chosen as a function of $\epsilon$. Due to (3.5), the frequency and the amplitude are even functions of $\epsilon$. Hence, $h$ should be an even function of $\epsilon$. For example, we can take $h=-\frac{1}{\sqrt{1+\gamma \epsilon^{2}}}$, where $\gamma$ is a positive constant. As $\gamma$ increases, the convergence regions of the amplitude and the frequency become larger and larger, as shown in Figs. 5 and 6.

We can integrate Eq. (1.1) by Runge-Kutta method in order to obtain the limit cycle and its properties. Table 1 shows the value of the amplitude $a_{R K}$ obtained by using Runge-Kutta method and the value obtained by homotopy-Padé technique (see [16]), where for briefly a few cases reported. Clearly, the amplitude converges to the exact value for various $\epsilon$.

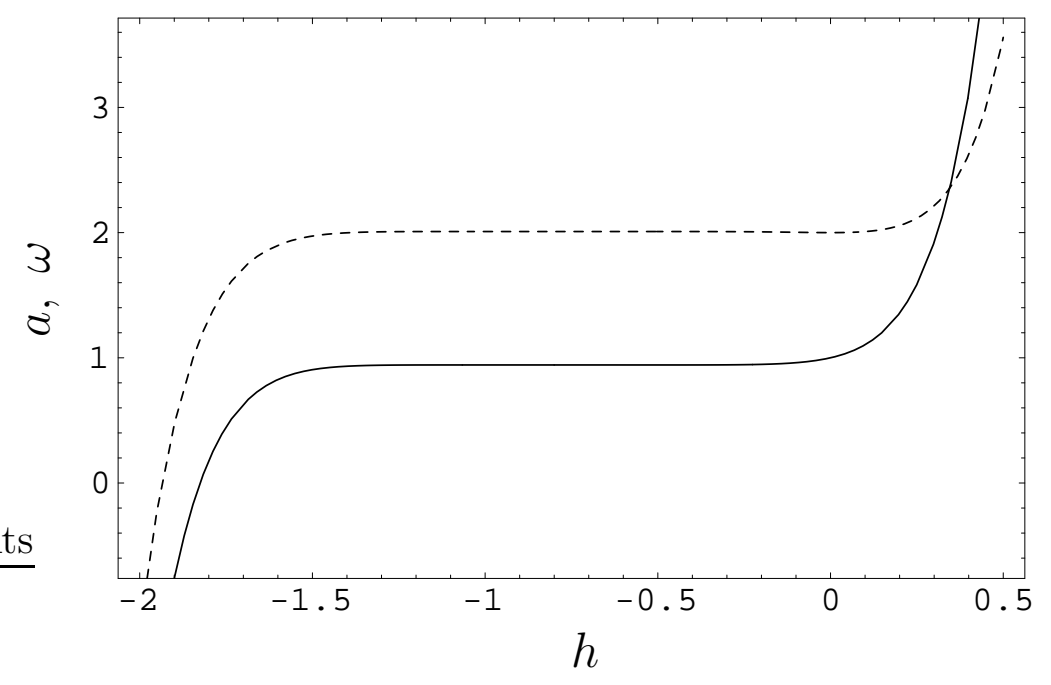

Fig. 1: The curves of the wave amplitude $a$ and frequency $\omega$ versus $h$ for the 10th-order approximation for $\epsilon=1$. Solid curve: the wave frequency; dotted line: the wave amplitude. 


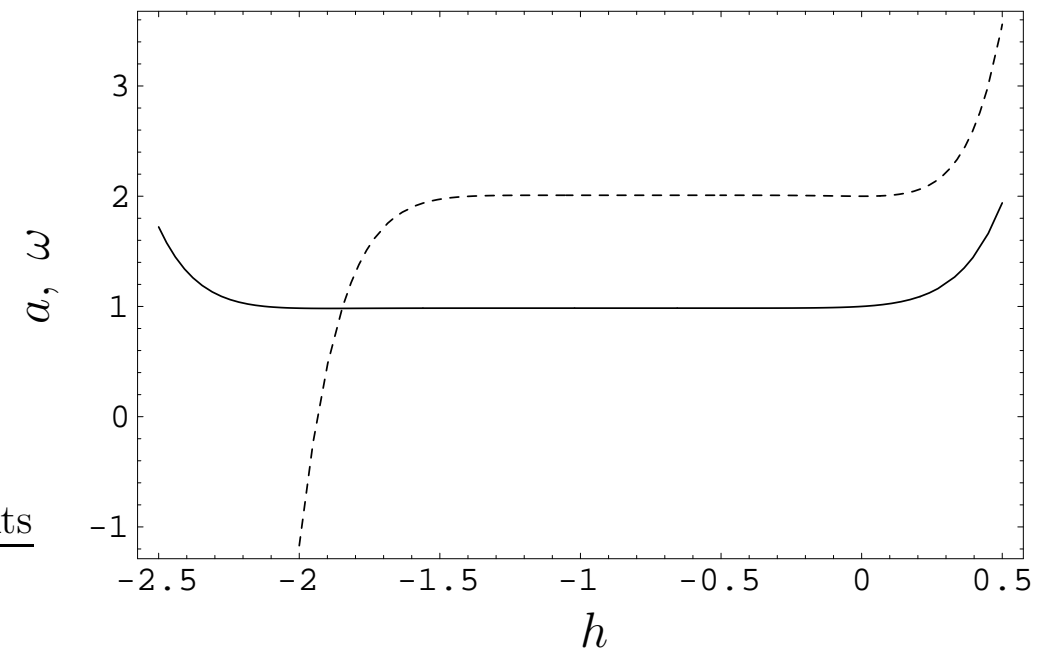

Fig. 2: The curves of the wave amplitude $a$ and frequency $\omega$ versus $h$ for the 10th-order approximation for $\epsilon=0.5$. Solid curve: the wave frequency; dotted line: the wave amplitude.

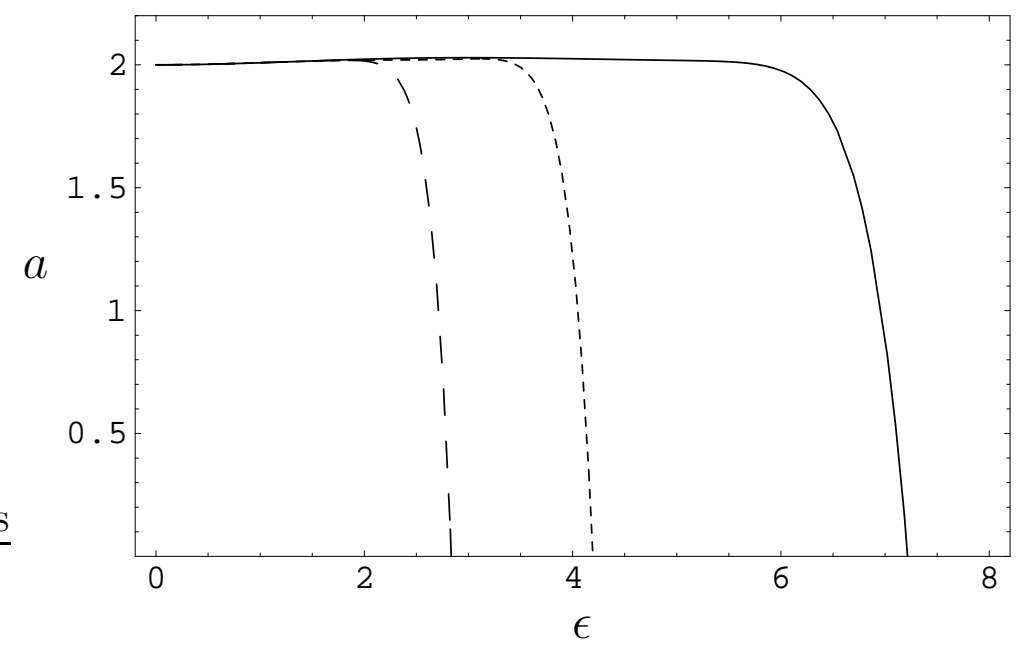

Fig. 3: Comparison of the amplitude of the 10th-order homotopy analysis approximation. Solid curve: $h=-\frac{1}{3}$, dotted curve: $h=-\frac{2}{3}$, dashed curve: $h=-1$. 


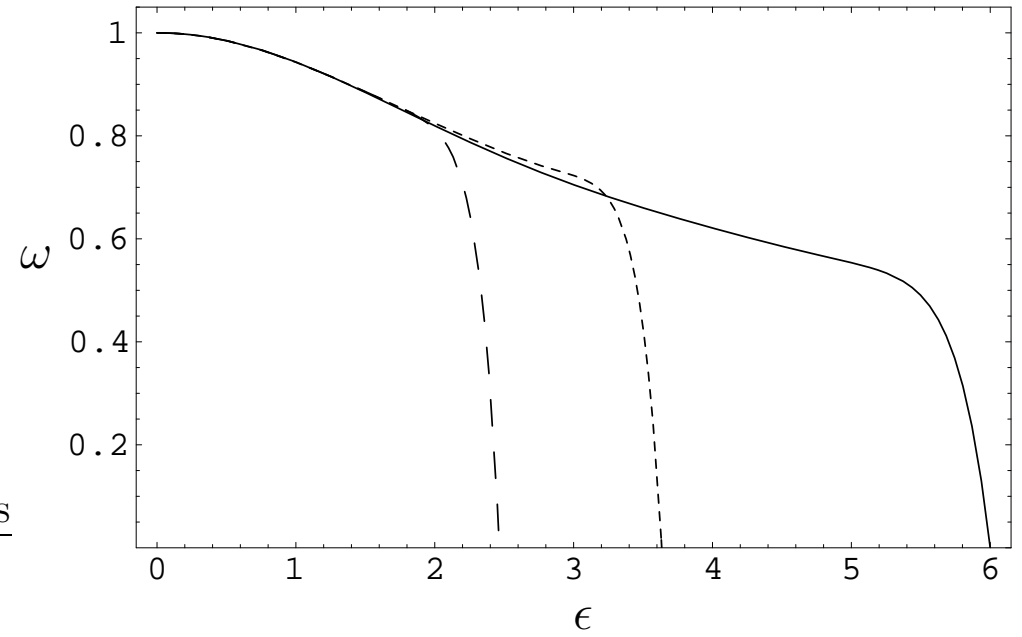

Fig. 4: Comparison of the frequency of the 10th-order homotopy analysis approximation. Solid curve: $h=-\frac{1}{3}$, dotted curve: $h=-\frac{2}{3}$, dashed curve: $h=-1$.

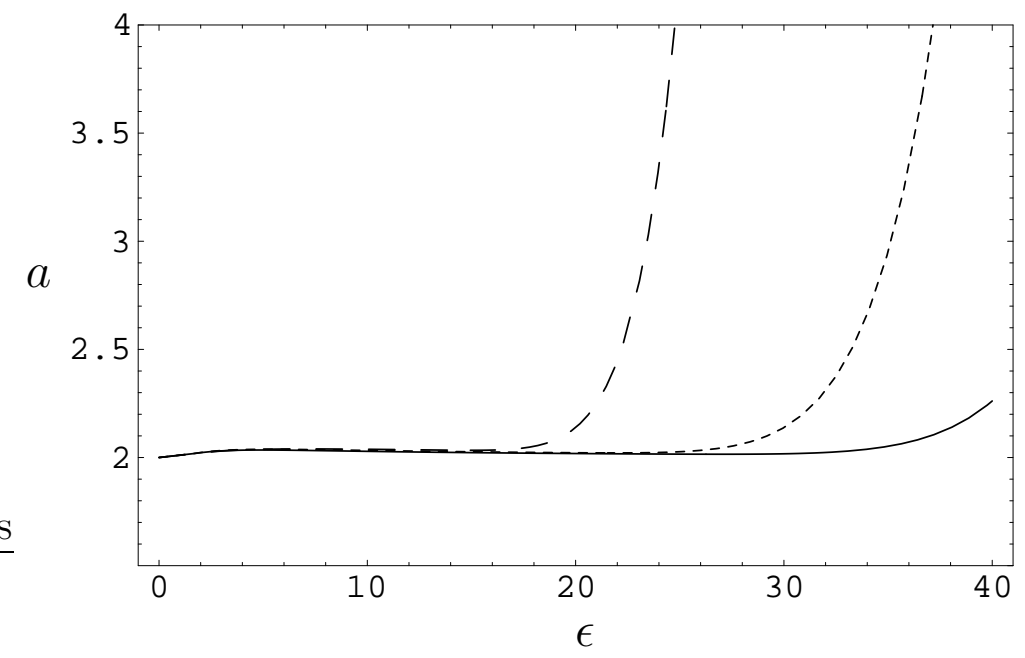

Fig. 5: Comparison of the amplitude of the 10th-order homotopy analysis approximation. Solid curve: $\gamma=3$, dotted curve: $\gamma=2$, dashed curve: $\gamma=1$. 


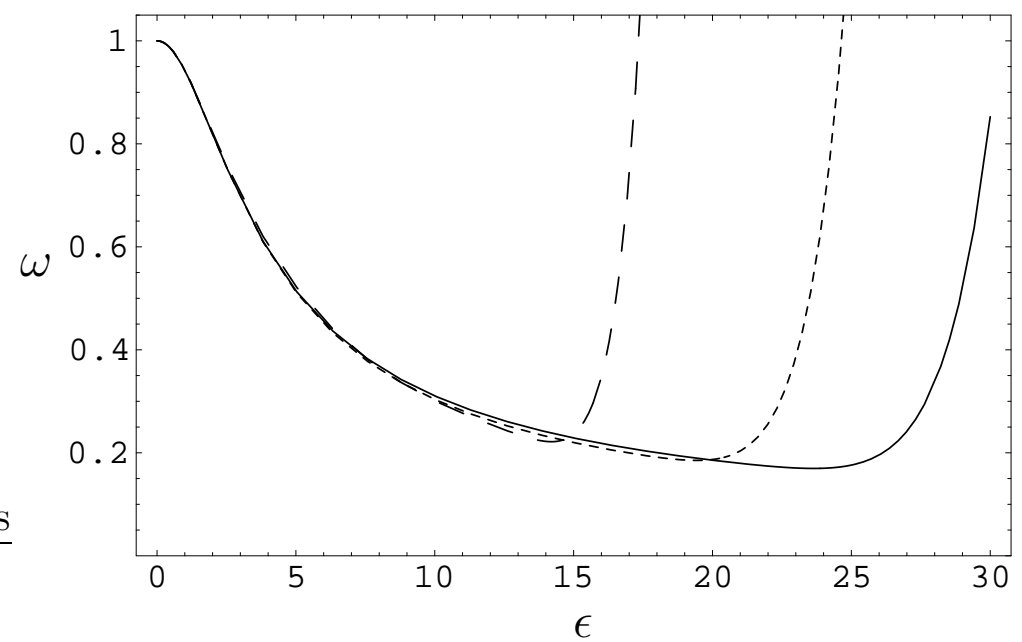

Fig. 6: Comparison of the frequency of the 10th-order homotopy analysis approximation. Solid curve: $\gamma=3$, dotted curve: $\gamma=2$, dashed curve: $\gamma=1$.

Table 1: Results for $[m, m]$ Homotopy-Padé approach for Example 1

\begin{tabular}{c|ccccccc}
\hline$\epsilon$ & 0.1 & 0.3 & 0.5 & 0.7 & 0.9 & 1.5 & 2.0 \\
\hline$a_{R K}$ & 2.00010 & 2.00092 & 2.00248 & 2.00466 & 2.00724 & 2.01523 & 2.01989 \\
{$[2,2]$} & 2.00010 & 2.00092 & 2.00249 & 2.00469 & 2.00737 & 2.01670 & 2.02426 \\
{$[3,3]$} & 2.00010 & 2.00092 & 2.00249 & 2.00469 & 2.00737 & 2.01670 & 2.02427 \\
{$[4,4]$} & 2.00010 & 2.00092 & 2.00249 & 2.00466 & 2.00724 & 2.01515 & 2.01943 \\
{$[5,5]$} & 2.00010 & 2.00092 & 2.00249 & 2.00466 & 2.00724 & 2.01514 & 2.01936 \\
{$[6,6]$} & 2.00010 & 2.00092 & 2.00249 & 2.00466 & 2.00724 & 2.01523 & 2.02001 \\
{$[7,7]$} & 2.00010 & 2.00092 & 2.00249 & 2.00466 & 2.00724 & 2.01523 & 2.02001 \\
{$[8,8]$} & 2.00010 & 2.00092 & 2.00249 & 2.00466 & 2.00724 & 2.01523 & 2.01989
\end{tabular}

Example 2. Here, we consider $f(x)=5 x^{4}-9 x^{2}+1$. This system has two limit cycles, one stable and the other one unstable [37].

The corresponding approximation of their amplitudes,

$$
\begin{aligned}
& a(\epsilon)=1.755170+0.017880 \epsilon^{2}+\mathcal{O}\left(\epsilon^{4}\right), \\
& \bar{a}(\epsilon)=0.720677+0.00390888 \epsilon^{2}+\mathcal{O}\left(\epsilon^{4}\right),
\end{aligned}
$$

by a recursive algorithm was reported in [8,13]. Under transformation (2.2), Eq. (1.1) becomes

$$
\omega^{2} u^{\prime \prime}(\tau)+\epsilon \omega\left[5 a^{4} u^{4}(\tau)-9 a^{2} u^{2}(\tau)+1\right] u^{\prime}(\tau)+u(\tau)=0 .
$$

From (2.17), the term $R_{m}(\tau)$ in (2.15) becomes 


$$
\begin{aligned}
R_{m}(\tau)= & \sum_{n=0}^{m-1} u_{m-1-n}^{\prime \prime}(\tau)\left(\sum_{j=0}^{n} \omega_{j} \omega_{n-j}\right)+u_{m-1}(\tau)+\epsilon \sum_{n=0}^{m-1} \omega_{n} u_{m-n-1}^{\prime}(\tau) \\
& +5 \epsilon \sum_{n=0}^{m-1}\left[\left(\sum_{i=0}^{m-1-n} \omega_{i} u_{m-n-i-1}^{\prime}(\tau)\right) \sum_{j=0}^{n}\left(\sum_{r=0}^{j} \hat{a}_{r} \hat{a}_{j-r}\right)\left(\sum_{s=0}^{n-j} \hat{u}_{s}(\tau) \hat{u}_{n-j-s}(\tau)\right)\right] \\
& -9 \epsilon \sum_{n=0}^{m-1}\left[\left(\sum_{i=0}^{m-1-n} \omega_{i} u_{m-n-i-1}^{\prime}(\tau)\right) \sum_{j=0}^{n}\left(\sum_{r=0}^{j} a_{r} a_{j-r}\right)\left(\sum_{s=0}^{n-j} u_{s}(\tau) u_{n-j-s}(\tau)\right)\right] .
\end{aligned}
$$

where

$$
\hat{a}_{n}=\sum_{i=0}^{n} a_{i} a_{n-i}, \quad \hat{u}_{n}(\tau)=\sum_{i=0}^{n} u_{i}(\tau) u_{n-i}(\tau) .
$$

It is found that the frequency $\omega$ and the amplitude $a$ at the $M$ th-order of approximation can be expressed in the form (3.5). So, $a_{0}$ and $\omega_{0}$ are obtained by solving (2.18) for $m=1$, i.e.

$$
c_{1,1}=\left(1-\omega_{0}^{2}\right)=0, \quad d_{1,1}=\epsilon \omega_{0}\left(8-18 a_{0}^{2}+5 a_{0}^{4}\right)=0 .
$$

Hence, we have two limit cycles with $\omega_{0}=1$ : one of them with amplitude $a_{0}=$ $\sqrt{\frac{9+\sqrt{41}}{5}}$ (stable limit cycle for $\epsilon>0$ ), and the other one with amplitude $\bar{a}_{0}=\sqrt{\frac{9-\sqrt{41}}{5}}$ (unstable limit cycle for $\epsilon>0$ ). The obtained results for the amplitude with $a_{0}$ as initial guess are as follows:

$$
\begin{aligned}
A_{1}= & 1.75517, \\
A_{2}= & 1.75517+0.0178803 h^{2} \epsilon^{2}, \\
A_{3}= & 1.75517+0.0536409 h^{2} \epsilon^{2}+0.0357606 h^{3} \epsilon^{2}+0.0151888 h^{3} \epsilon^{4}, \\
A_{4}= & 1.75517+0.107282 h^{2} \epsilon^{2}+0.143042 h^{3} \epsilon^{2}+0.0536409 h^{4} \epsilon^{2}+0.0607553 h^{3} \epsilon^{4} \\
& -0.179337 h^{4} \epsilon^{4}+0.0129025 h^{4} \epsilon^{6} .
\end{aligned}
$$

For the frequency are

$$
\begin{aligned}
\Omega_{1}= & 1+0.424737 h \epsilon^{2}, \\
\Omega_{2}= & 1+0.849473 h \epsilon^{2}+0.424737 h^{2} \epsilon^{2}+0.270602 h^{2} \epsilon^{4}, \\
\Omega_{3}= & 1+1.27421 h \epsilon^{2}+1.27421 h^{2} \epsilon^{2}+0.424737 h^{3} \epsilon^{2}+0.811805 h^{2} \epsilon^{4} \\
& +0.451679 h^{3} \epsilon^{4}+0.191558 h^{3} \epsilon^{6}, \\
\Omega_{4}= & 1+1.69895 h \epsilon^{2}+2.54842 h^{2} \epsilon^{2}+1.69895 h^{3} \epsilon^{2}+0.424737 h^{4} \epsilon^{2}+1.62361 h^{2} \epsilon^{4} \\
& +1.80672 h^{3} \epsilon^{4}+0.543231 h^{4} \epsilon^{4}+0.76623 h^{3} \epsilon^{6}+0.38455 h^{4} \epsilon^{6}+0.142383 h^{4} \epsilon^{8} .
\end{aligned}
$$

In particular, for $h=-1$, in 10th-order approximation we obtain

$$
\begin{aligned}
A_{10}= & 1.75517+0.0178803 \epsilon^{2}-0.240092 \epsilon^{4}+0.859582 \epsilon^{6}-0.227156 \epsilon^{8}-13.7118 \epsilon^{10} \\
& +10.9555 \epsilon^{12}+4.73704 \epsilon^{14}-0.626997 \epsilon^{16}+0.00484811 \epsilon^{18}+\mathcal{O}\left(\epsilon^{20}\right) .
\end{aligned}
$$

And for $h=-1$, in 10th-order approximation with $\bar{a}_{0}$ as initial guess, we have 


$$
\begin{aligned}
\bar{A}_{10}= & 0.720677+0.00390888 \epsilon^{2}-0.000410295 \epsilon^{4}-0.0000165055 \epsilon^{6} \\
& +9.1144410^{-6} \epsilon^{8}-1.4504510^{-7} \epsilon^{10}-2.4244510^{-7} \epsilon^{12}-1.4559310^{-7} \epsilon^{14} \\
& +9.9172410^{-10} \epsilon^{16}+5.3716810^{-11} \epsilon^{18}+\mathcal{O}\left(\epsilon^{20}\right) .
\end{aligned}
$$

We can investigate the influence of $h$ on the convergence of $a$ and $\omega$ by plotting the curve of $a$ and $\omega$ versus $h$, as shown in Fig. 7. One can see that, for $\epsilon=0.5$, we have $-0.9 \leq h \leq-0.2$. The comparison of the amplitude $a$ and the frequency $\omega$ at the 10th-order of approximation with the numerical results is as shown in Figs. 8 and 9 , where $h=-1,-\frac{2}{3}$ and $-\frac{1}{3}$. However, as $h$ is negative and close to zero, the convergence region becomes larger and larger, as in Example 1.

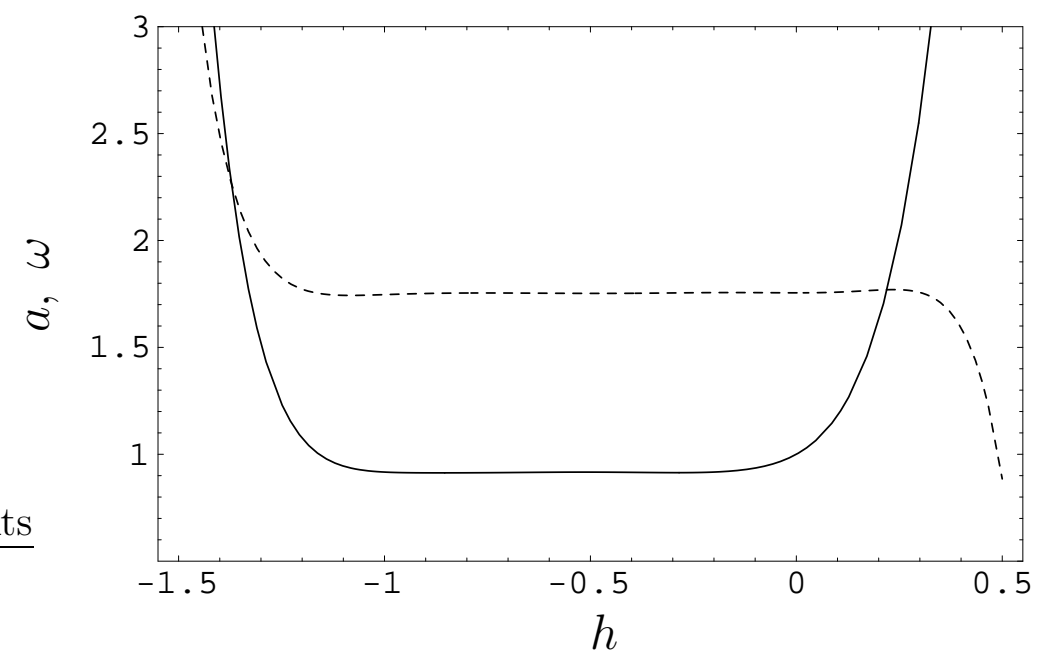

Fig. 7: The curves of the wave amplitude $a$ and frequency $\omega$ versus $h$ for the 10th-order approximation for $\epsilon=0.5$. Solid curve: the wave frequency; dotted line: the wave amplitude.

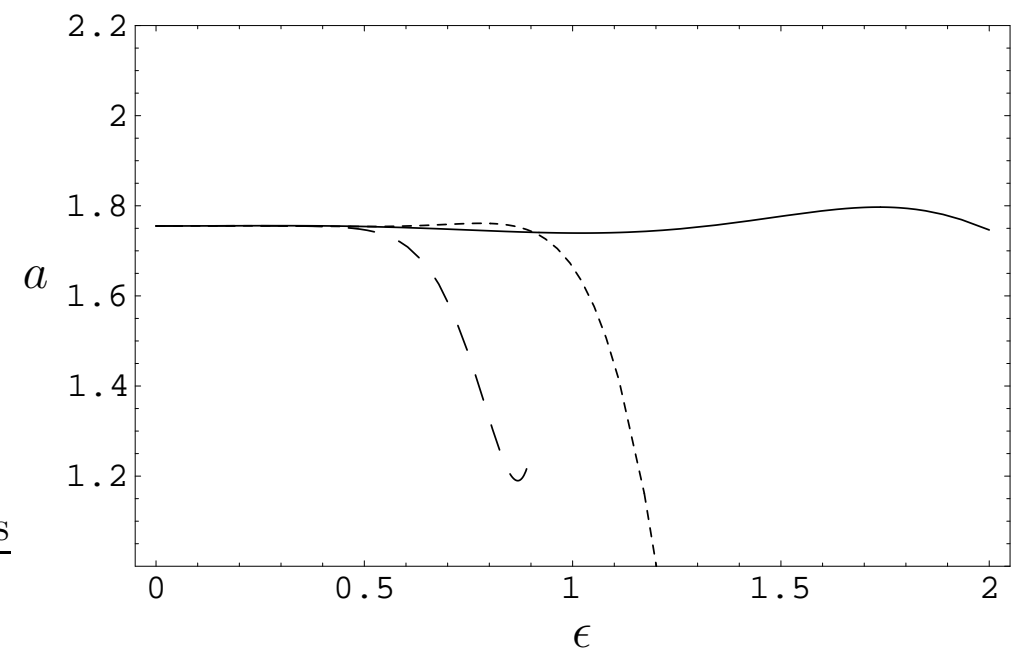

Fig. 8: Comparison of the amplitude of the 10th-order homotopy analysis approximation. Solid curve: $h=-\frac{1}{3}$, dotted curve: $h=-\frac{2}{3}$, dashed curve: $h=-1$. 


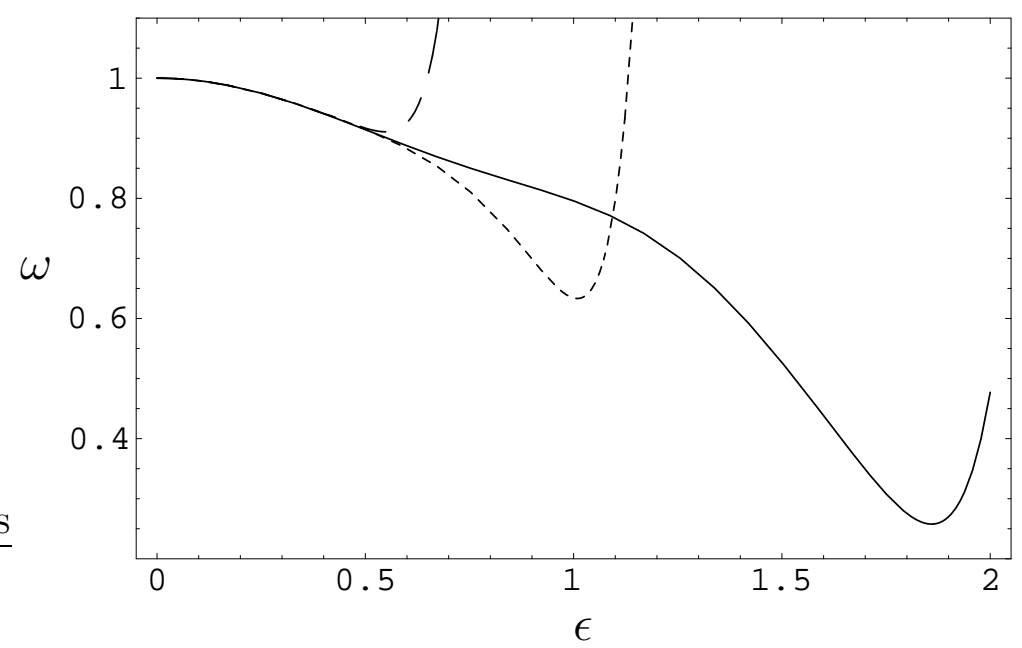

Fig. 9: Comparison of the frequency of the 10th-order homotopy analysis approximation. Solid curve: $h=-\frac{1}{3}$, dotted curve: $h=-\frac{2}{3}$, dashed curve: $h=-1$.

\section{Conclusions}

We have applied the homotopy analysis method (HAM) to the classical Liénard differential equation (1.1) to obtain analytic approximations of the amplitude and frequency of its limit cycles. Two examples have been explicitly worked out. The results obtained with the HAM are in excellent agreement with the known solutions. Moreover, the HAM provides us with a convenient way (the parameter $h$ ) to control the convergence of approximation series; this is a fundamental qualitative difference between the HAM and other methods for finding approximate solutions. In particular, the case $h=-1$ corresponds with the exact perturbative expansion in $\epsilon$.

Let us conclude by saying that the examples shown in this paper are illustrative of the power of the HAM to solve complicated nonlinear problems.

\section{References}

[1] Andronov AA, Vitt AA, Khaikin SE. Theory of oscillators, Dover, New York; 1989.

[2] Odani K. The limit cycle of the van der Pol equation is not algebraic. J Differen Equat 1995;115:146-152.

[3] López-Ruiz R, Pomeau Y. Transition between two oscillation modes. Phys Rev E 1997; 55:R3820-R3823.

[4] Ye Y. Theory of limit cycles. In: Trans Math Monographs, vol. 66. Boston: American Mathematical Society; 1986. 
[5] Lins A, de Melo W, Pugh CC. On Liénards equation. In: Lectures Notes in Math, vol. 597. Springer-Verlag; 1977. p. 355.

[6] Rychkov GS. The maximum number of limit cycles of the system $\dot{y}=-x, \dot{x}=$ $y-\sum_{i=0}^{2} a_{i} x^{2 i+1}$ is two. Differen Equat 1975;11:301-302.

[7] López JL, López-Ruiz R. The limit cycles of Liénard equations in the strongly nonlinear regime. Chaos, Solitons \& Fractals 2000;11:747-756.

[8] López JL, López-Ruiz R. Approximating the amplitude and form of limit cycles in the weakly nonlinear regime of Liénard systems. Chaos, Solitons \& Fractals 2007;34:13071317.

[9] Dumortier D, Panazzolo D, Roussarie R. More limit cycles than expected in the Liénard equations. Proc Am Math Soc 2007;135:1895-1904.

[10] Giacomini H, Neukirch S. Number of limit cycles of the Liénard equation. Phys Rev E 1997; 56:3809-3813.

[11] Verhulst, F. Nonlinear differential equations and dynamical equations, SpringerVerlag, Berlin; 1990.

[12] Anderson C, Geer J. Power series expansions for the frequency and period of the limit cycle of the van der Pol equation. SIAM J Appl Math 1982;42:678-693.

[13] López JL, López-Ruiz R. The limit cycles of Liénard equations in the weakly nonlinear regime. ArXiv 2006:nlin/0605025.

[14] Depassier MC, Mura J. Variational approach to a class of nonlinear oscillators with several limit cycles. Phys Rev E 2001;64:056217(6).

[15] Liao SJ. The proposed homotopy analysis technique for the solutions of non-linear problems, Ph.D. Thesis, Shanghai Jiao Tong University, 1992.

[16] Liao SJ. Beyond perturbation: introduction to the homotopy analysis method. Boca Raton: Chapman \& Hall/CRC Press; 2003.

[17] Liao SJ. An analytic approximate approach for free oscillations of self-excited systems. Int J Non-Linear Mech 2004;39:271-280.

[18] Abbasbandy S. The application of homotopy analysis method to solve a generalized Hirota-Satsuma coupled KdV equation. Phys Lett A 2007;361:478-483.

[19] Abbasbandy S. Homotopy analysis method for heat radiation equations. Int Commun Heat Mass Transf 2007;34:380-387.

[20] Abbasbandy S, Tan Y, Liao SJ. Newton-Homotopy analysis method for nonlinear equations. Appl Math Comput 2007;188:1794-1800.

[21] Abbasbandy S, Samadian Zakaria F. Soliton solutions for the fifth-order KdV equation with the homotopy analysis method. Nonlinear Dynam 2008;51:83-87.

[22] Abbasbandy S. Solitary wave solutions to the Kuramoto-Sivashinsky equation by means of the homotopy analysis method. Nonlinear Dynam 2008;52:35-40. 
[23] Abbasbandy S. Soliton solutions for the Fitzhugh-Nagumo equation with the homotopy analysis method. Appl Math Model (in press).

[24] Liao SJ. A new branch of solutions of boundary-layer flows over an impermeable stretched plate. Int J Heat Mass Transfer 2005;48:2529-2539.

[25] Liao SJ. Series solutions of unsteady boundary-layer flows over a stretching flat plate. Stud Appl Math 2006;117:239-264.

[26] Liao SJ, Magyari E. Exponentially decaying boundary layers as limiting cases of families of algebraically decaying ones. Z Angew Math Phys (ZAMP) 2006;57:777792 .

[27] Liao SJ, Su J, Chwang AT. Series solutions for a nonlinear model of combined convective and radiative cooling of a spherical body. Int J Heat Mass Transfer 2006;49:2437-2445.

[28] Tan Y, Xu H, Liao SJ. Explicit series solution of travelling waves with a front of Fisher equation. Chaos, Solitons \& Fractals 2007;31:462-472.

[29] Wu W, Liao SJ. Solving solitary waves with discontinuity by means of the homotopy analysis method. Chaos, Solitons \& Fractals 2005;26:177-185.

[30] Hayat T, Khan M. Homotopy solutions for a generalized second-grade fluid past a porous plate. Nonlinear Dynam 2005;42:395-405.

[31] Hayat T, Khan M, Ayub M. On non-linear flows with slip boundary condition. Z Angew Math Phys (ZAMP) 2005;56:1012-1029.

[32] Liao SJ, Cheung K. Homotopy analysis of nonlinear progressive waves in deep water. J Eng Math 2003;45:105-116.

[33] Sajid M, Hayat T, Asghar S. On the analytic solution of the steady flow of a fourth grade fluid. Phys Lett A 2006;355:18-26.

[34] Tan Y, Abbasbandy S. Homotopy analysis method for quadratic Riccati differential equation. Commun Nonlinear Sci Numer Simul 2008;13:539-546.

[35] Wang C. Analytic solutions for a liquid film on an unsteady stretching surface. Heat Mass Transfer 2006;42:759-766.

[36] Abbasbandy S, Parkes EJ. Solitary smooth-hump solutions of the Camassa-Holm equation by means of the homotopy analysis method. Chaos, Solitons \& Fractals 2008;36:581-591.

[37] López-Ruiz R, López JL. Bifurcation curves of limit cycles in some Liénard systems. Int J Bifurcat Chaos 2000;10:971-980. 